\title{
Guiding properties of large mode area silicon microstructured fibers: a route to effective single mode operation
}

\author{
N. Vukovic, N. Healy, and A. C. Peacock* \\ Optoelectronics Research Centre, University of Southampton, Southampton, SO17 1BJ, UK \\ *Corresponding author: acp@orc.soton.ac.uk
}

Received March 4, 2011; revised April 25, 2011; accepted April 27, 2011; posted April 27, 2011 (Doc. ID 143681); published May 24, 2011

Numerical simulations are used to investigate the guiding properties of large mode area silicon microstructured fibers. Modal analysis of the isolated high refractive index core and cladding rod inclusions will be applied to show that the guidance mechanism of the composite fiber can be well described via a hybrid of the total internal reflection and antiresonant reflecting optical waveguide models. It will be shown that by selectively filling the cladding holes with silicon, which has been modified to have a slightly raised index, the fiber can be designed to operate in an effectively single-mode regime over an extended wavelength range. (c) 2011 Optical Society of America

OCIS codes: $\quad 060.2280,060.2290,160.6000,190.4370$

\section{INTRODUCTION}

Silicon photonics is fast becoming one of the most active areas of research with applications ranging from optical interconnects, to nonlinear signal processing [1], through to biosensors [2]. Much of the progress made in this area has leveraged off the highly developed silicon-on-insulator (SOI) platform, where the high index contrast between the core and cladding results in strong mode confinement so that waveguides can be scaled to submicrometer dimensions. Unfortunately, however, integrating the rectilinear nanoscale devices with micrometer-sized cylindrical fiber platforms requires complex coupling arrangements, and damage thresholds typically limit the optical power that can be launched into the small scale cores [3]. A potential solution to these problems that is currently generating much interest is to incorporate the active semiconductor component into the fiber geometry to yield a new class of silicon core optical fibers [느무. Fiberized silicon devices offer some notable advantages over those developed on-chip, such as simple, low-cost fabrication, robust and versatile waveguide geometries, as well as extended electromagnetic interaction lengths.

To date, a number of silicon fibers have been fabricated via various techniques where typically the semiconductor core is clad in silica so that they are analogous to the SOI waveguides. However, an alternative geometry that allows for greater control of the waveguide properties are the silicon-silica microstructured optical fibers (SSMOFs), as demonstrated in []]. This SSMOF was fabricated via the complete filling of a hollow core pure silica photonic bandgap guiding fiber (PBGF) with the semiconductor material using a high-pressure chemical deposition technique. The resultant structure consisted of a high-index silicon core with a cladding defined by a lattice of silicon rods embedded in the low-index silica webbing. Despite having a large $\sim 9 \mu \mathrm{m}$ diameter core, this fiber was shown to only support two core modes over an extended telecoms wavelength range, either of which could be prefer- entially excited via modifying the launch conditions. As well as offering improved coupling with standard silica fibers and higher-power handling capabilities, this large mode area (LMA) SSMOF displayed similar properties to the more conventional air-silica LMA MOFs. Importantly, a key feature of the LMA MOFs is that they can be designed to exhibit effective single-mode guidance, which is a critical requirement for many signal processing and nonlinear applications.

In this paper we will extend the previous investigations of the mode analysis of the LMA SSMOF to better understand the guidance properties with the aim to achieving effective singlemode operation. Previous investigations on microstructured fibers that have high-index inclusions embedded in a lowindex interconnected cladding have shown that the guidance mechanism can be best described via a hybrid of the total internal reflection (TIR) and antiresonant reflecting optical waveguide (ARROW) models [7-9]. Following a similar analysis, we will show that the mode properties of the SSMOF can be understood via coupling between the modes of the highindex core with those in the high-index cladding rods. Using this picture, a method of selectively filling the cladding holes with a slightly higher-index material than the core will be proposed to isolate the fundamental mode for preferential excitation. We anticipate that SSMOFs with effective single-mode guidance could find use in a number of all-optical processing devices (e.g., demultiplexing, reshaping, retiming), which are important for high-capacity, high-speed optical communication systems.

\section{DESCRIPTION OF THE GUIDANCE MECHANISM OF SSMOFS}

The SSMOFs on which our numerical investigations are based are fabricated by completely filling the PBGF template shown in Fig. 1(a), where the central core hole is defined by omitting three cells from an hexagonal lattice. The important structural parameters that define the cladding are the hole diameter 

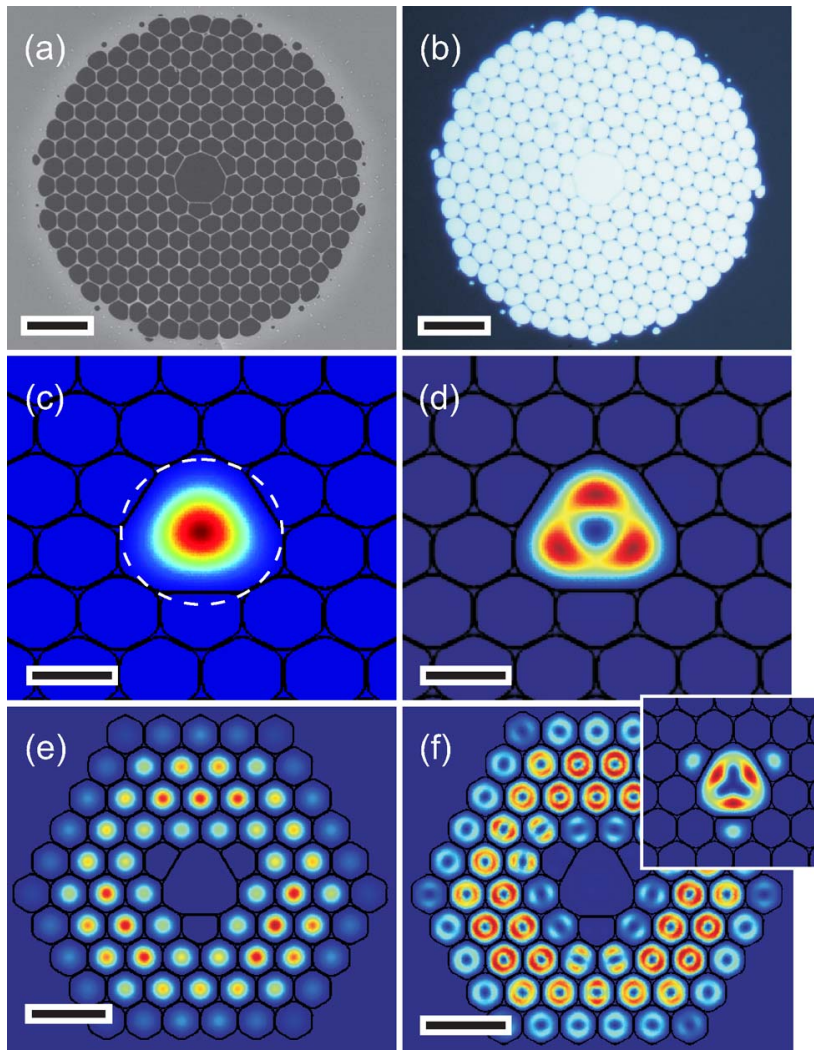

Fig. 1. (Color online) (a) Silica PBGF template and (b) SSMOF; scale bars $10 \mu \mathrm{m}$. (c) Fundamental and (d) second-order core modes at $1.55 \mu \mathrm{m}$; scale bars $4 \mu \mathrm{m}$ [dashed line in (c) is equivalent circular core of the SSMOF]. (e) Fundamental and (f) second-order cladding modes at $1.55 \mu \mathrm{m}$; scale bars $8 \mu \mathrm{m}$. Inset, a higher-order mode that exists below the first cladding band.

( $d=4.2 \mu \mathrm{m}$ ), the hole-to-hole spacing (pitch, $\Lambda=4.33 \mu \mathrm{m}$ ), and the hole roundedness $\left(d_{c} / d=0.5\right)$, where $d_{c}$ is the diameter of curvature at the corners that defines the hexagonal shape of the inclusions [10]. A fabricated SSMOF is shown in Fig. 1(b), where the low-index PBG guiding core has been converted to a high-index waveguide. This SSMOF has an equivalent circular core diameter of $8.7 \mu \mathrm{m}$ [see dashed curve in Fig. 1(c)], similar to a standard single-mode fiber, and it is important to note that a step-index silicon core, silica cladding fiber with these dimensions would support more than a thousand core-guided modes [11]. Nevertheless, even though the silicon core is completely embedded in the silica webbing, experimentally only two modes could be excited in this fiber over a wavelength range of $1.3-1.7 \mu \mathrm{m}$, and these modes could be preferentially coupled via modifying the launch conditions.

To better understand the guidance properties of this fiber we have extended the previous full vector finite element method (FEM) modeling. Because of the triangular shape of the core, the full structure is modeled and anisotropic perfectly matched layers are positioned outside the outermost ring of holes in order to reduce the simulation window [12] (which introduced a small imaginary component to the mode indices of the order $10^{-18}$ ). For convenience, we have based our modeling on the parameters of single crystal silicon as the wavelength dependence of the refractive index is well documented [13]. Figures $\underline{1(\mathrm{c})}$ and $\underline{1(\mathrm{~d})}$ show the fundamental and second-order core modes that are supported by the SSMOF, below which there exists a broad band of cladding modes.
Figure 1(e) shows the first cladding mode, which is similar to the fundamental space filling mode used to define the effective cladding index $n_{\text {clad }}=n_{\text {FSM }}$ of a conventional air-silica MOF [14]. Searching below this cladding mode, however, we find that there are further TIR-guided core modes in the SSMOF that are then followed by a second band of higherorder cladding modes as illustrated in Fig. 1(f). The positioning of cladding modes between the higher-order core-guided modes [an example of which is shown in the inset of Fig. 1(f)] indicates that the TIR guidance mechanism has been modified by the presence of the cladding rods.

Further insight into the nature of the guiding mechanisms can be provided by comparing the mode properties of the full SSMOF structure with those of the isolated triangular core and a single hexagonal cladding rod. In all cases the modal analysis is conducted via FEM modeling as described above with the same sized computational windows, but with the triangular core and hexagonal rod surrounded by a pure silica cladding. Figure 2(a) shows the index profile of the SSMOF together with the calculations of the mode indices for the three structures at $1.55 \mu \mathrm{m}$. From this we see that the core modes of the SSMOF (solid lines) are positioned at similar, but slightly higher indices than those of the isolated step-index core (gray dashed), and that the first cladding band of the SSMOF (light blue) is introduced precisely at the position of the fundamental mode of an individual cladding rod (dashed black). This coupling of light from the core to the
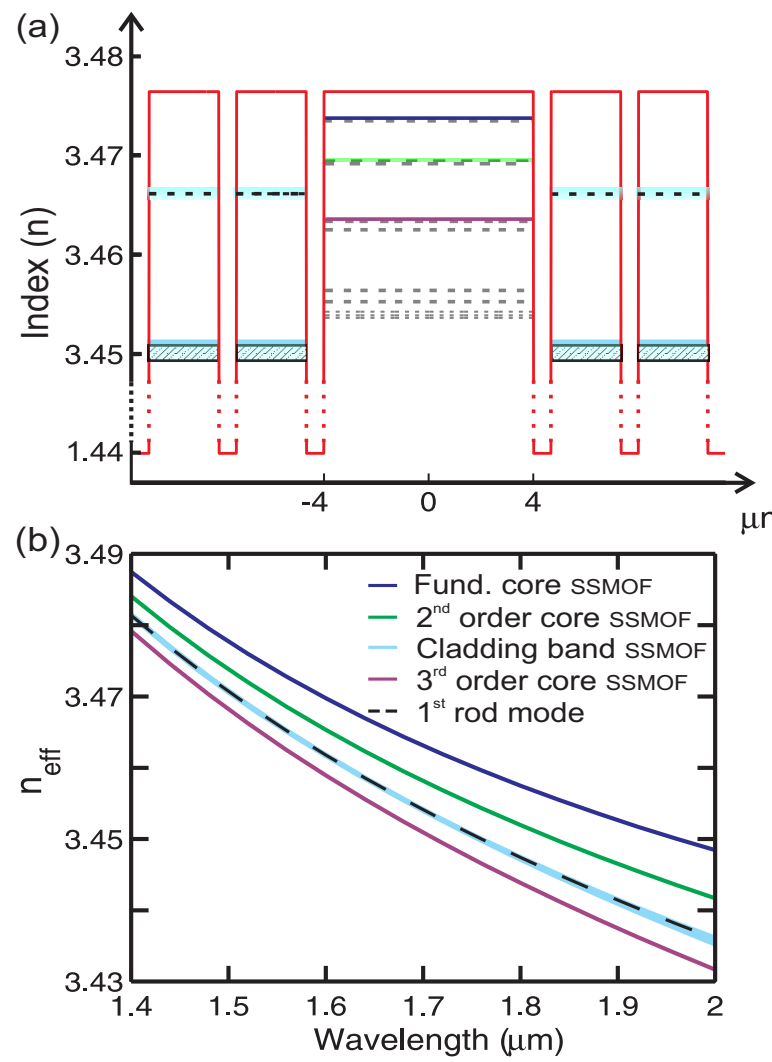

Fig. 2. (Color online) (a) SSMOF index profile and the calculated mode indices at $1.55 \mu \mathrm{m}$. Isolated core (gray dashed) and cladding rod modes (black dashed) are plotted together with the modes of the full SSMOF (solid lines); colors correspond to the legend in (b). (b) Wavelength dependence of the full SSMOF mode indices (solid) and the fundamental mode of a cladding rod (dashed). 
cladding rods as observed here is characteristic of the ARROW guiding mechanism [8], and the cladding profiles seen in Figs. 1(e) and 1(f) thus exhibit the characteristic shapes of the fundamental and second-order rod modes, respectively. We note that the presence of the TIR-guided core modes below the cladding bands is analogous to the coreguided modes that exist below $n_{\text {clad }}$ in air-silica LMA MOFs [14]. As the higher-order core modes of the SSMOF are essentially lossless bound modes they cannot be discounted without further consideration, much like the higher-order core modes of the LMA MOFs, which can be associated with low confinement losses. However, while such higher-order modes have been observed in meter lengths of MOF, despite many efforts we have not succeeded in exciting them in the SSMOFs. A possible explanation for this is that in practice the loss value of the second-order mode in the SSMOF is more than twice that of the fundamental (as measured in [6]), which we attribute both to the silicon material loss and the increased interaction of the second-order mode with the cladding. This suggests that the loss of the higher-order core modes will be even greater, which would render them invisible. Nevertheless, despite their known existence, even in the MOFs these higher-order modes are typically difficult to launch into [14] and, thus, they are most often ignored. Furthermore, given the relatively short lengths required for device operation in silicon core fibers (typically of the order of centimeters), it is unlikely that conversion to these modes will occur during transmission.

In this fiber, the positioning of the fundamental cladding band is significant as it isolates the two lowest-order core modes, which, in practice, allows for their preferential excitation. Figure 2(b) shows the effective indices of the three lowest-order SSMOF modes and the first cladding band over the wavelength range $1.4-2 \mu \mathrm{m}$. From this it is clear that the dispersion of this cladding follows that of the fundamental rod mode, and that it lies between the second- and third-order modes over this wavelength span. In fact, the modeling indicates that this cladding band remains above the third-order modes over the entire silicon low-loss transmission window so that the SSMOF is effectively dual-moded. Thus, from these results is evident that in order to modify the guiding properties of this SSMOF to shift the first cladding band above the second-order core mode, the index of the fundamental cladding rod modes must be raised.

\section{SELECTIVELY FILLED SSMOFS}

From Fig. 2(a), it is clear that there are two obvious routes to increasing the fundamental mode index of the cladding rods: (i) increase the rod size, or (ii) raise the material index of the rod. As the starting PBGF template already has an air-filling fraction of $93 \%$, only a very small increase in the rod size would be possible, insufficient to shift the fundamental cladding mode index above the second-order mode in the core. However, the index of silicon can be tuned simply via the introduction of dopants (to either crystalline [15] or amorphous materials [16]) and there are a number of techniques that allow for the selective filling of cladding holes in MOFs [9,17]. Importantly, the high-pressure chemical deposition technique used to fabricate the SSMOF in Fig. 1(b) can be easily controlled via the choice of temperature, precursor concentration, and flow rate, to tune the deposited material param- eters, making it well-suited to this approach. It is useful to note that, as the fundamental modes of the SSMOFs are well confined to the core, the introduction of potentially lossy cladding materials (e.g., doped silicon) will have little effect on the propagation losses of the SSMOFs.

Figure 3(a) shows the index profile of a selectively filled SSMOF (SF-SSMOF) when the material index of the cladding rods is raised by $d n_{\text {rod }}=0.006$ at $1.55 \mu \mathrm{m}$. This is plotted together with the first two mode indices of the isolated core (gray dashed) and those of individual cladding rods with $d n_{\text {rod }}$ of 0.006 (black dashed), 0.004 (purple), and 0.002 (yellow), where the small values of $d n_{\text {rod }}$ are within the range achievable via material doping. These results show that, at this wavelength, raising the index by $d n_{\text {rod }}=0.004$ should be sufficient to position the first cladding band between the fundamental and second-order core modes. However, extending the modeling over the $1.4-2 \mu \mathrm{m}$ wavelength range, at longer wavelengths $(>1.7 \mu \mathrm{m})$ the index of this mode shifts below the second-order core mode. Thus, in order to ensure that the fundamental mode remains isolated over this entire range, it is necessary to raise the index of the cladding rods by $d n_{\text {rod }}=$ 0.006 . This is confirmed via calculating the mode indices for the full SF-SSMOF structure, as shown as the solid curves in Fig. 3(b). For comparison, Fig. 3(b) also shows the fundamen-

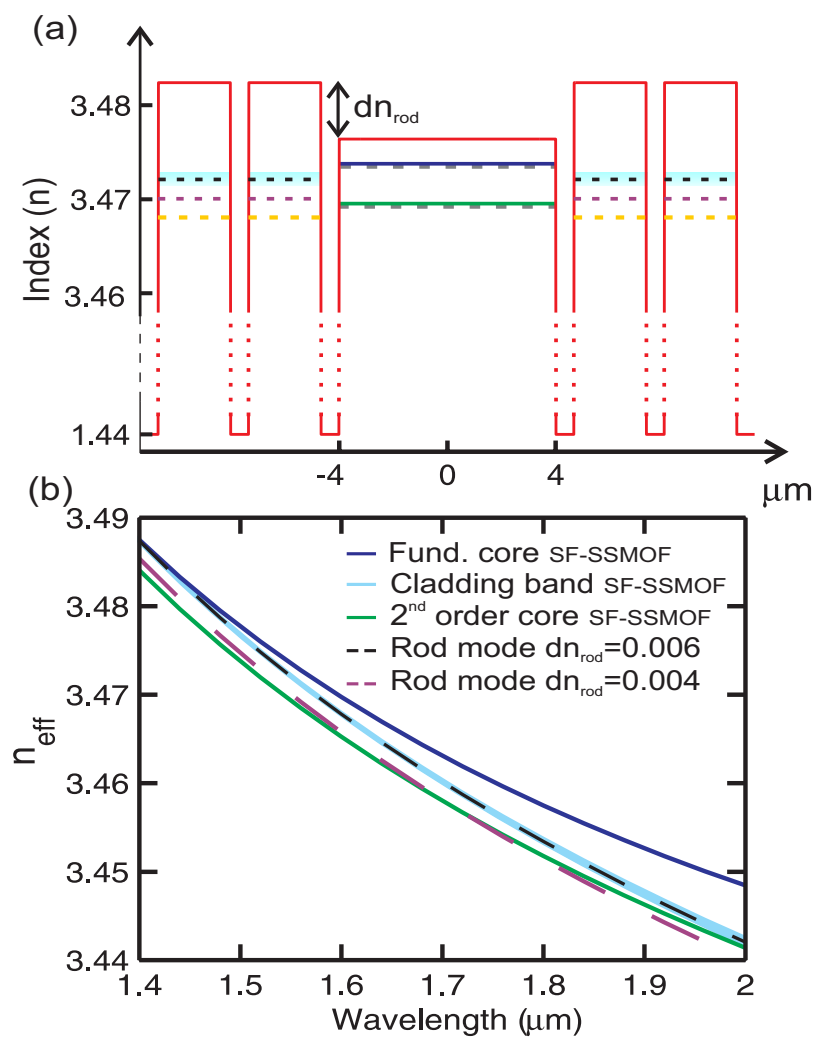

Fig. 3. (Color online) (a) SF-SSMOF index profile and the isolated core modes (gray dashed), together with the fundamental modes of the raised index cladding rods: $d n_{\text {rod }}=0.006$ (black dashed, top), 0.004 (purple, middle), 0.002 (yellow, bottom) at $1.55 \mu \mathrm{m}$. Solid lines are the modes of the full SF-SSMOF in (b) to show that they are still well approximated by those of the isolated core and cladding rods. (b) Wavelength dependence of the full SF-SSMOF mode indices with $d n_{\text {rod }}=0.006$, compared to the curves for the fundamental rod modes, as labeled in the legend. 


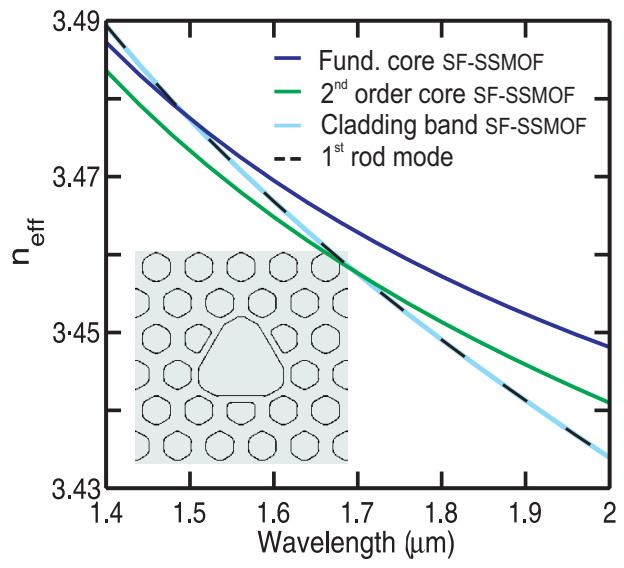

Fig. 4. (Color online) Wavelength dependence of the mode indices for the full SF-SSMOF with $d=2.8 \mu \mathrm{m}$ and a raised cladding rod index of $d n_{\text {rod }}=0.018$. Inset shows the PBGF template structure.

tal rod mode with $d n_{\text {rod }}=0.004$ (purple dashed) to verify that this shifts below the second-order core mode of the SFSSMOF (green solid), which is a good approximation to the second-order mode of the isolated core [as shown via the comparison in Fig. 3(a)]. We note that the difference in the slopes of the index curves for the core and cladding rod modes is due to their different sizes, where the smaller rods have their dispersion altered by a larger waveguide contribution.

Finally, although it is not practical to consider increasing the inclusion size in the PBGF template used here, it is possible to reduce the rod size, which will thus require a further increase in the cladding rod material index to isolate the fundamental core mode. Figure $\underline{4}$ shows the effective mode indices of a SF-SSMOF with a reduced hole diameter $d=$ $2.8 \mu \mathrm{m}$ and a raised cladding rod index of $d n_{\text {rod }}=0.018$. As these smaller rods experience an even larger waveguide contribution to their dispersion (shown as the dashed black line), the cladding band now crosses both the fundamental and second-order modes within this wavelength range. We note that this phase matching allows for coupling from the core to the cladding modes, which has been used to suppress modes in both TIR [8] and PBG guiding fibers [18]. Thus, by positioning the indices of the cladding mode in this fashion, we predict that it will still be possible to isolate the fundamental mode in the SF-SSMOFs over a reduced wavelength range $1.5-1.7 \mu \mathrm{m}$. It is interesting to note that, unlike in PBGFs, the cutting on and off of the TIR core modes in these hybrid fibers does not dramatically alter the dispersion of the modes, as evident from the continuous effective index curves in Fig. 4, making their guiding properties quite unique $[\underline{8}, \underline{9}, \underline{19}]$.

\section{CONCLUSION}

We have investigated the modal properties of SSMOFs to show that the guidance mechanism can be well described via a hybrid of the TIR and ARROW models. By simply comparing the effective mode indices of the isolated core and cladding rods, it is possible to design SF-SSMOFs with carefully positioned cladding bands to reduce the number of modes that can be effectively excited in the core. Importantly, this analysis can be applied to fibers filled with different semiconductor materials, including higher-index core materials, such as germanium, which when clad in pure silica can be highly multimode even for submicrometer core dimensions. The abil- ity to design large mode area semiconductor waveguides that facilitate integration with existing fiber infrastructures, but yet can still offer effectively single-mode operation, has great potential to reduce complexity and improve device efficiency in a wide range of applications.

\section{ACKNOWLEDGMENTS}

The authors acknowledge the Engineering and Physical Sciences Research Council (EPSRC) and the National Science Foundation (NSF) Materials World Network for financial support and thank J. R. Sparks for the fabrication of the siliconsilica microstructured fiber and P. Horak and F. Poletti for helpful discussions. A. C. Peacock holds a Royal Academy of Engineering fellowship.

\section{REFERENCES}

1. B. Jalali and S. Fathpour, "Silicon photonics," J. Lightwave Technol. 24, 4600-4615 (2006).

2. A. Densmore, D.-X. Xu, S. Janz, P. Waldron, T. Mischki, G. Lopinski, A. Delâge, J. Lapointe, P. Cheben, B. Lamontagne, and J. H. Schmid, "Spiral-path high-sensitivity silicon photonic wire molecular sensor with temperature-independent response," Opt. Lett. 33, 596-598 (2008).

3. M. Lipson, "Overcoming the limitations of microelectronics using Si nanophotonics: solving the coupling, modulation and switching challenges," Nanotech. 15, S622-S627 (2004).

4. L. Lagonigro, N. Healy, J. R. Sparks, N. F. Baril, P. J. A. Sazio, J. V. Badding, and A. C. Peacock, "Low loss silicon fibers for photonics applications," Appl. Phys. Lett. 96, 041105 (2010).

5. J. Ballato, T. Hawkins, P. Foy, R. Stolen, B. Kokuoz, M. Ellison, C. McMillen, J. Reppert, A. M. Rao, M. Daw, S. Sharma, R. Shori, O. Stafsudd, R. R. Rice, and D. R. Powers, "Silicon optical fiber," Opt. Express 16, 18675-18683 (2008).

6. N. Healy, J. R. Sparks, M. N. Petrovich, P. J. A. Sazio, J. V. Badding, and A. C. Peacock, "Large mode area silicon miscrostructured fiber with robust dual mode guidance," Opt. Express 17, 18076-18082 (2009).

7. N. M. Litchinitser, S. C. Dunn, B. Usner, B. J. Eggleton, T. P. White, R. C. McPhedran, and C. M. de Sterke, "Resonances in microstructured optical waveguides," Opt. Express 11, 1243-1251 (2003).

8. L. Lavoute, P. Roy, A. Desfarges-Berthelemot, V. Kermène, and S. Février, "Design of microstructured single-mode fiber combining large mode area and high rare earth ion concentration," Opt. Express 14, 2994-2999 (2006).

9. N. Healy, J. R. Sparks, R. He, P. J. A. Sazio, J. V. Badding, and A. C. Peacock, "High index contrast semiconductor ARROW and hybrid ARROW fibers," Opt. Express, doc. ID 144221 (2011, in press).

10. M. N. Petrovich, F. Poletti, A. van Brakel, and D. J. Richardson, "Robustly single mode hollow core photonic bandgap fiber," Opt. Express 16, 4337-4346 (2008).

11. A. W. Snyder and J. D. Love, Optical Waveguide Theory (Chapman and Hall, 1983).

12. K. Saitoh, M. Koshiba, T. Hasegawa, and E. Sasaoka, "Chromatic dispersion control in photonic crystal fibers: application to ultra-flattened dispersion," Opt. Express 11, 843-852 (2003)

13. H. H. Li, "Refractive index of silicon and germanium and its wavelength and temperature derivatives," J. Phys. Chem. Ref. Data 9, 561-658 (1980).

14. J. C. Flanagan, R. Amezcua, F. Poletti, J. R. Hayes, N. G. R. Broderick, and D. J. Richardson, "The effect of periodicity on the defect modes of large mode area microstructured fibers," Opt. Express 16, 18631-18645 (2008).

15. R. A. Soref and B. R. Bennett, "Electrooptical effects in silicon," IEEE J. Quantum Electron. 23, 123-129 (1987).

16. G. Cocorullo, F. D. Corte, R. De Rosa, I. Rendina, A. Rubino, and E. Terzini, "Amorphous silicon-based guided-wave 
passive and active devices for silicon integrated optoelectronics," IEEE J. Sel. Top. Quantum Electron. 4, 997-1002 (1998).

17. Y. Huang, Y. Xu, and A. Yariv, "Fabrication of functional microstructured optical fibers through a selective-filling technique," Appl. Phys. Lett. 85, 5182-5184 (2004).
18. T. Murao, K. Saitoh, and M. Koshiba, "Multiple resonant coupling mechanism for suppression of higher-order modes in all-solid photonic bandgap fibers with heterostructured cladding," Opt. Express 19, 1713-1727 (2011).

19. C.-P. Yu and J.-H. Liou, "Selectively liquid-filled photonic crystal fibers for optical devices," Opt. Express 17, 8729-8734 (2009). 